\title{
2013 Gastrointestinal Cancers Symposium: meeting highlights
}

Manisha Palta', Christopher Willett ${ }^{1} \&$ Brian Czito*1 $^{* 1}$

\section{Gastrointestinal Cancers Symposium, 24-26 January 2013, Moscone West Building, San Francisco, CA, USA}

The Gastrointestinal Cancers Symposium is an annual meeting geared toward physicians and researchers interested in the prevention, detection and treatment of gastrointestinal malignancies including medical, surgical and radiation oncologists, radiologists, gastroenterologists, pathologists and translational-oriented laboratory scientists.

The Gastrointestinal Cancers Symposium is a 3-day meeting covering a logical progression of topics from the upper to lower gastrointestinal (GI) tract - Thursday: cancers of the esophagus and stomach; Friday: cancers of the pancreas, small bowel and hepatobiliary tract; and Saturday: cancers of the colon and rectum. Each day begins with a session on prevention, screening and diagnosis, followed by a session focusing on translational research and selected oral abstracts and concluding with sessions on the multidisciplinary treatment of respective disease sites. The primary focus of this conference scene is to highlight notable data from cancers of the colon and rectum, as well as practice-changing data from other disease sites pertinent to GI oncology.

\section{Colorectal cancer}

Two trials in metastatic colorectal cancer (CRC) further clarified optimal chemotherapy (CT) regimens. The Italian TRIBE study randomized 508 previously untreated patients to 5-fluorouracil (5FU), leucovorin, oxaliplatin, irinotecan (FOLFOXIRI)/bevacizumab (bev) versus
5FU, leucovorin, irinotecan (FOLFIRI)/ bev. The primary end point, progressionfree survival (PFS), was improved from a median PFS of 9.5 months with FOLFIRI/ bev to 11.9 months with FOLFOXFIRI/ bev $(p=0.001)$. Grade $3 / 4$ GI toxicities were similar in both regimens with slightly higher rates of hematologic toxicities in the FOLFOXIRI/bev cohort [1]. A second multinational European trial evaluated the role of bev in addition to capecitabine (cap) in a relatively understudied population, the elderly. The AVEX trial randomized 280 patients $\geq 70$ years old to cap versus cap/bev. Median age at enrollment was 76 and the primary end point was PFS. Patients receiving cap/bev had a significant increase in PFS of 9.1 months compared with 5.1 months with cap alone. A nonsignificant improvement in median overall survival (OS) was seen with cap/bev: 20.7 versus 16.8 months. As expected, rates of arterial and venous thromboembolism were nearly double with bev (3 and $8 \%$, respectively). AVEX represents the first randomized trial evaluating the role of bev in elderly patients with metastatic CRC

'Department of Radiation Oncology, Duke University Medical Center, NC, USA

*Author for correspondence: brian.czito@duke.edu

News \& Views

News

Journal Watch

Interview

Conference Scene
Future 
[2]. These trials indicate that the addition of new agents to standard CT regimens is associated with lower rates of disease progression with reasonable toxicity rates.

Unlike the robust data of the role of systemic therapy in the treatment of patients with metastatic CRC, the role of adjuvant CT in stage II colon cancer remains unclear. Common clinical practice is to recommend adjuvant CT for 'high-risk' stage II patients with one of the following characteristics: obstruction or perforation, T4 stage, fewer than 12 lymph nodes excised, involved surgical margins, lymphovascular invasion or perineural invasion. Data from the British Columbia Cancer Registry evaluated 1697 stage II patients treated between 1999-2008 (1236 high risk and 461 low risk). While most patients did not receive adjuvant CT, 29\% of highrisk patients received adjuvant treatment. Individuals receiving adjuvant CT were younger with a better performance status. After adjusting for confounding variables, a statistically significant survival advantage was seen in patients with high-risk features receiving adjuvant CT: 5-year OS: 75\% with adjuvant CT compared with 68\% with no adjuvant CT. However, relapsefree survival and disease-specific survival benefits were seen in T4 patients suggesting a limited role for CT in 'high-risk' patients and possible trend towards harm in a lowrisk group receiving CT [3]. Retrospective analyses such as these are limited by patient selection.

For patients with stage II-III rectal cancer the standard of care throughout most of the USA and parts of Europe is neoadjuvant, long-course chemoradiation. A number of studies have indicated superior outcomes in patients who achieve a pathologic complete response (pCR), with some studies suggesting that patients achieving a complete clinical response may, with close surveillance, forego surgical resection [4,5]. Efforts to optimize neoadjuvant regimens to obtain higher $\mathrm{pCR}$ rates have been the focus of ongoing study as reflected in a number of abstracts from this year's meeting. A Phase II study from Brown University (RI, USA) reported on the feasibility of delivering all planned CT in the neoadjuvant setting and its impact on $\mathrm{pCR}$ and complete resection (R0). Of a planned 39 patients with locally advanced rectal cancer, 36 received modified FOLFOX6 every 2 weeks for eight cycles, followed by chemoradiation (CRT) with 5-FU-based CT and surgical resection within 4-8 weeks. A total of 26 patients completed all planned preoperative therapy with 21 patients undergoing surgical resection. R0 resections were achieved in $100 \%$ of patients with six (29\%) demonstrating pCR [6]. A second Phase II study reported on 37 of a planned 55 patients with locally advanced rectal cancer, delivering a neoadjuvant regimen of oxaliplatin, capecitabine and celecoxib with concurrent radiotherapy (RT). A total of 21 patients underwent surgery with complete pathologic evaluation. Of these, seven (33\%) had a pCR [7]. Phase III data with the addition of oxaliplatin to 5FU-based CRT have shown no significant improvement in pCR or clinical outcomes with higher rates of toxicities; the effect of celecoxib and other agents concurrent with radiation may warrant further investigation [8-10].

As emerging data has shown short-term equivalence of neoadjuvant, long-course CRT and short-course RT in terms of local control in rectal cancer patients, some neoadjuvant studies are utilizing a shortcourse approach for RT delivery [11,12]. A Phase II study of 80 patients with cT3-T4 rectal cancer from Washington University, USA initiated neoadjuvant treatment with short-course RT (5 Gy $\times$ five fractions) followed by four cycles of mFOLFOX6. Of 76 evaluable patients, 21 (28\%) achieved a pCR, with only $9 \%$ experiencing grade 3 GI toxicity during neoadjuvant therapy [13].

\section{Noncolorectal cancer}

Clinical research in GI malignancies has focused on the integration of targeted agents into therapy, particularly EGF receptor (EGFR) and VEGF inhibitors, which are involved in tumorogenesis and metastasis development. An important lesson reiterated in this year's meeting is that an agent that has been shown to be efficacious in the metastatic setting does not necessarily translate to improved outcomes in patients with localized disease. Data in the metastatic setting show improved outcomes 
with the addition of trastuzumab (a monoclonal antibody against human HER2/ EGFR2) to standard CT for patients with advanced gastric or gastroesophageal junction cancers exhibiting HER2 overexpression [14]. In head and neck squamous cancers, the addition of cetuximab to RT resulted in a significant improvement in OS [15]. Nearly $80 \%$ of esophageal adenocarcinomas and squamous cell carcinomas overexpress EGFR and hence inhibition of this pathway may represent an important therapeutic target [16].

A multicenter Phase II/III trial from the UK randomized 258 patients with esophageal cancer with plans for definitive CRT with cisplatin/capecitabine to treatment with or without cetuximab. The Phase II primary end point, 24-week failure-free survival in the cetuximab arm, was not met and the trial was closed on the basis of futility. Patients receiving cetuximab experienced higher rates of nonhematologic toxicities and lower rates of standard treatment completion. A significant detriment to median OS was seen with the addition of cetuximab (22 vs 25 months) [17]. Results from this study prompted the suspension of recruitment to RTOG 0436, a randomized study evaluating the addition of cetuximab to paclitaxel, cisplatin and RT in patients with esophageal cancer treated without surgery. In pancreatic cancer, an interim analysis of an ongoing Phase III Japanese trial comparing adjuvant $S 1$ versus gemcitabine in resected pancreatic cancer patients was reported. $\mathrm{S} 1$ is an oral fluoropyrimidine that combines tegafur, gimeracil and oteracil, and has previously been shown to enhance survival in Japanese patients undergoing gastric cancer resection $[18,19]$. In the pancreatic study, a preplanned interim analysis after the first 205 deaths (in 385 patients) showed that $70 \%$ of $S 1$ patients were alive at 2 years follow-up compared with 51\% receiving gemcitabine, with corresponding 2-year disease-free progression rates of 49 versus $29 \%$, respectively [20]. In patients with locally advanced pancreatic cancer, a randomized Phase II study of induction CT followed by gemcitabine versus capecitabine-based chemoradiation was reported. Patients received three cycles of gemcitabine/capecitabine CT initially and those with stable/responding disease, tumor diameter $\leq 6 \mathrm{~cm}$ and favorable performance status received a further cycle of the same CT and were then randomized to either capecitabine-based CRT versus gemcitabine-based CRT. Primary study end point was 9-month PFS. Grade 3/4 hematologic and nonhematological toxicity was higher in the gemcitabine arm, with a 9-month PFS (63 vs 51\%) and OS (15.2 vs 13.4 months) improved in the capecitabine-containing arm [21]. In patients with metastatic pancreatic cancer, a randomized Phase III study of weekly nab-paclitaxel plus gemcitabine versus gemcitabine alone (the MPACT study) was reported. Nab-paclitaxel is an albumin-bound drug that provides tumor-selective localization via transcytosis across the endothelium, potential tumor uptake via macropinocytosis and improved pharmacokinetics versus unbound paclitaxel, while also demonstrating increased tumoral gemcitabine levels in vitro. In this study of 861 patients OS (8.5 vs 6.7 months median), PFS (5.5 vs 3.7 months median) and time to treatment failure (5.1 vs 3.6 months median) were significantly improved in the nab-paclitaxel-containing arm [22].

\section{Conclusion}

Few conferences address prevention, diagnosis, treatment and management of patients with GI malignancies in a true multidisciplinary forum. Data presented at this year's conference will continue to help shape and guide contemporary treatment approaches as well as facilitate future breakthroughs in gastrointestinal cancers.

\section{Financial \& competing interests}

\section{disclosure}

The authors have no relevant affiliations or financial involvement with any organization or entity with a financial interest in or financial conflict with the subject matter or materials discussed in the manuscript. This includes employment, consultancies, honoraria, stock ownership or options, expert testimony, grants or patents received or pending, or royalties.

No writing assistance was utilized in the production of this manuscript. 


\section{References}

1 Loupakis F, Cremolini C, Masi G et al. FOLFOXIRI plus bevacizumab (bev) versus FOLFIRI plus bev as first-line treatment of metastatic colorectal cancer (MCRC): Results of the Phase III randomized TRIBE trial. J. Clin. Oncol. 30(Suppl. 34), Abstract 336 (2012).

2 Cunningham D, Lang I, Lorusso $\mathrm{V}$ et al. Bevacizumab (bev) in combination with capecitabine (cape) for the first-line treatment of elderly patients with metastatic colorectal cancer (mCRC): Results of a randomized international Phase III trial (AVEX). J. Clin. Oncol. 30(Suppl. 34), Abstract 337 (2012).

3 Kumar A, Kennecke HF, Lim HJ et al. Use of adjuvant chemotherapy (AC) and outcomes in stage II colon cancer (CC) with versus without poor prognostic features. J. Clin. Oncol. 30(Suppl. 34), Abstract 338 (2012).

4 Maas M, Beets-Tan RG, Lambregts DM et al. Wait-and-see policy for clinical complete responders after chemoradiation for rectal cancer. J. Clin. Oncol. 29, 4633-4640 (2011).

5 Habr-Gama A, Perez RO, Nadalin W et al. Operative versus nonoperative treatment for stage 0 distal rectal cancer following chemoradiation therapy: long-term results. Ann. Surg. 240, 711-717; discussion 717-718 (2004).

6 Perez K, Pricolo V, Vrees M et al. A Phase II study of complete neoadjuvant therapy in rectal cancer (CONTRE): The Brown University Oncology Group. J. Clin. Oncol. 30(Suppl. 34), Abstract 335 (2012).

7 Murad W, Fekrazad HM, Schrader R et al. A Phase II trial of combination oxaliplatin, capecitabine, and celecoxib with concurrent radiation for patients with newly diagnosed resectable rectal cancer. J. Clin. Oncol. 30(Suppl. 34), Abstract 487 (2012).

8 Aschele C, Cionini L, Lonardi S et al. Primary tumor response to preoperative chemoradiation with or without oxaliplatin in locally advanced rectal cancer: pathologic results of the STAR-01 randomized Phase III trial. J. Clin. Oncol. 29, 2773-2780 (2011).

9 Roh MS, Yothers G, O'Connell MJ et al. The impact of capecitabine and oxaliplatin in the preoperative multimodality treatment in patients with carcinoma of the rectum: NSABP R-04. J. Clin. Oncol. 29, Abstract 3503 (2011).

10 Gerard JP, Azria D, Gourgou-Bourgade S et al. Clinical outcome of the ACCORD 12/0405 PRODIGE 2 randomized trial in rectal cancer. J. Clin. Oncol. 30, 4558-4565 (2012).

11 Bujko K, Nowacki MP, NasierowskaGuttmejer A et al. Long-term results of a randomized trial comparing preoperative short-course radiotherapy with preoperative conventionally fractionated chemoradiation for rectal cancer. Br. J. Surg. 93, 1215-1223 (2006).

12 Ngan SY, Burmeister B, Fisher RJ et al. Randomized trial of short-course radiotherapy versus long-course chemoradiation comparing rates of local recurrence in patients with T3 rectal cancer: trans-tasman radiation oncology group trial 01.04. J. Clin. Oncol. 30, 3827-3833 (2012).

13 Myerson RJ, Parikh PJ, Hunt SR et al. Effect of short course radiation followed by four cycles of FOLFOX as preoperative therapy for rectal cancer on rate of PCR: a Phase II trial. J. Clin. Oncol. 30(Suppl. 34), Abstract 471 (2012).

14 Bang YJ, Van Cutsem E, Feyereislova A et al. Trastuzumab in combination with chemotherapy versus chemotherapy alone for treatment of HER2-positive advanced gastric or gastro-oesophageal junction cancer (ToGA): a Phase 3, open-label, randomised controlled trial. Lancet 376, 687-697 (2010).

15 Bonner JA, Harari PM, Giralt J et al. Radiotherapy plus cetuximab for locoregionally advanced head and neck cancer: 5 year survival data from a Phase 3 randomised trial, and relation between cetuximab-induced rash and survival. Lancet Oncol. 11, 21-28 (2010).

16 Takaoka M, Harada H, Andl CD et al. Epidermal growth factor receptor regulates aberrant expression of insulin-like growth factor-binding protein 3. Cancer Res. 64, 7711-7723 (2004).

17 Crosby T, Hurt C, Falk S et al. SCOPE 1: a Phase II/III trial of chemoradiotherapy in esophageal cancer plus or minus cetuximab. J. Clin. Oncol. 30(Suppl. 34), Abstract LBA3 (2012).

18 Sakuramoto S, Sasako M, Yamaguchi T et al. Adjuvant chemotherapy for gastric cancer with S-1, an oral fluoropyrimidine. $N$. Engl. J. Med. 357, 1810-1820 (2007).

19 Sasako M, Sakuramoto S, Katai $\mathrm{H}$ et al. Five-year outcomes of a randomized Phase III trial comparing adjuvant chemotherapy with S-1 versus surgery alone in stage II or III gastric cancer. J. Clin. Oncol. 29, 4387-4393 (2011).

20 Uesaka K, Fukutomi A, Boku N et al. Randomized Phase III trial of adjuvant chemotherapy with gemcitabine versus S-1 for patients with resected pancreatic cancer (JASPAC-01 study). J. Clin. Oncol. 30(Suppl. 34), Abstract 145 (2012).

21 Mukherjee S, Hurt C, Griffiths G et al. SCALOP: Results of a randomized Phase II study of induction chemotherapy followed by gemcitabine $(\mathrm{G})$ or capecitabine (Cap) based chemoradiation (CRT) in locally advanced pancreatic cancer (LANPC). J. Clin. Oncol. 30(Suppl. 34), Abstract LBA146 (2012).

22 Von Hoff DD, Ervin TJ, Arena FP et al. Randomized Phase III study of weekly nab-paclitaxel plus gemcitabine versus gemcitabine alone in patients with metastatic adenocarcinoma of the pancreas (MPACT). J. Clin. Oncol. 30(Suppl. 34), Abstract LBA148 (2012). 